\title{
Two-loop radiative seesaw with multicomponent dark matter explaining the possible $\gamma$ excess in the Higgs boson decay and at the Fermi LAT
}

\author{
Mayumi Aoki, ${ }^{1,2, *}$ Jisuke Kubo, ${ }^{1, \oplus}$ and Hiroshi Takano, ${ }^{1,}$ \\ ${ }^{1}$ Institute for Theoretical Physics, Kanazawa University, Kanazawa 920-1192, Japan \\ ${ }^{2}$ Max-Planck-Institut für Kernphysik, \\ Saupfercheckweg 1, 69117 Heidelberg, Germany
}

\begin{abstract}
A non-supersymmetric model of a two-loop radiative seesaw is proposed. The model contains, in addition to the standard model (SM) Higgs boson, an inert $S U(2)_{L}$ doublet scalar $\eta$ and two inert singlet scalars $\phi$ and $\chi$. The lepton number is softly broken by a dimension-two operator, and the tree-level mass of the left-handed neutrino is forbidden by $Z_{2} \times Z_{2}^{\prime}$ (or $D_{2 N}$ ), which predicts the existence of two or three dark matter particles. The scalar sector is minimal; none of the scalar fields can be suppressed for the radiative seesaw mechanism to work. There are by-products: The SM Higgs boson decay into two $\gamma^{\prime}$ 's is slightly enhanced by $\eta^{+}$(the charged component of $\eta$ ) circulating in one-loop diagrams for $h \rightarrow \gamma \gamma$. The $135 \mathrm{GeV} \gamma$-ray line observed at the Fermi LAT can be also explained by the annihilation of $\chi$ dark matter. We employ a mechanism of temperature-dependent annihilation cross section to suppress the continuum $\gamma$ rays and the production of antiprotons. The explanation can survive even down to the XENON1T sensitivity limit.
\end{abstract}

PACS numbers: $95.35 .+\mathrm{d}, 95.85 . \mathrm{Pw}, 11.30 . \mathrm{Er}$

*Electronic address: mayumi@hep.s.kanazawa-u.ac.jp

${ }^{\dagger}$ Electronic address: jik@hep.s.kanazawa-u.ac.jp

${ }^{\ddagger}$ Electronic address: takano@hep.s.kanazawa-u.ac.jp 


\section{INTRODUCTION}

Why the neutrino masses are small is a long-standing mystery. The seesaw mechanism [1 3] is an approach to provide an answer to it. The traditional seesaw mechanism indicates the existence of a super-high-scale physics beyond the standard model (SM). Another way to confront this problem is to generate the neutrino masses radiatively [4 6]. Many models have been proposed on the basis of the radiative generation of the neutrino masses, and the idea of the radiative seesaw mechanism [7, 8] is along the line of this idea: Right-handed neutrinos are introduced, but the Dirac masses are forbidden by a discrete symmetry. This discrete symmetry can be an origin of stable dark matter (DM) particles [7 11] in the Universe.

To produce the neutrino masses, the lepton number $\mathrm{L}$ has to be violated. In most of the models the lepton number $\mathrm{L}$ is violated softly by dimension-three operators, Majorana masses or scalar trilinear couplings, and the number of the loops ranges from one to three (see Refs. [12, 13] for different models). For the radiative seesaw mechanism, the number of loops $\ell$ means a scaling down of $(1+3 \ell)$ orders of magnitude for the right-handed neutrino mass (see also the discussions of Ref. [14]); $\left(k / 16 \pi^{2}\right)^{\ell} \simeq(k / 0.1) \times 6.3 \times 10^{-4},(k / 0.1)^{2} \times$ $4.0 \times 10^{-7}, \quad(k / 0.1)^{3} \times 2.5 \times 10^{-10}$ for $\ell=1,2$ and 3 , respectively, where $k$ is a generic coupling. Since the Majorana mass of the right-handed neutrino for the tree-level seesaw is $O\left(10^{10}\right) \mathrm{GeV}$, we may obtain a Majorana mass of $O(1) \mathrm{TeV}$ naturally in two-loop radiative seesaw models [15 17].

In this paper we propose a radiative seesaw model, in which the lepton number is softly broken by a dimension-two operator, and the neutrino masses are generated at the two-loop level. The discrete symmetry is $Z_{2} \times Z_{2}^{\prime}$ (or $D_{2 N}$ with $N=2,3, \ldots{ }^{1}$ ). Therefore, two or three DM particles can exist in this model ${ }^{2}$, which is a slight extension of the Ma model [8]. Obviously, radiative generation of the neutrino masses means an extension of the SM Higgs sector, which may have impacts on the existing experiments. In our model, we have a set of an inert doublet scalar $\eta$ and two singlet scalars. This set is minimal in the sense that the radiative neutrino mass generation does not work if one of them is suppressed. So, none of the extra scalar fields is ad hoc introduced.

Another motivation to extend the original Ma model is the following: If a neutral component of $\eta$ in the Ma model should be a realistic DM particle, its mass should be between 60 and $80 \mathrm{GeV}$ or larger than $500 \mathrm{GeV}$ [22, 23] (unless one allows very fine tuning of parameters [24]). In Ref. [21] we have slightly modified the Ma model such that there exist more than two stable DM particles and have found that the $\eta$ DM mass is then allowed to lie in a much wider range. But two scalars have been ad hoc added.

The existence of additional scalar doublets can change the decay rates of the SM Higgs

\footnotetext{
${ }^{1} D_{2 N}$ (the dihedral group of order $2 N$ ) is larger than $Z_{2} \times Z_{2}^{\prime}$. However, we use only the one-dimensional representations of $D_{2 N}$ so that $D_{2 N}$ acts as $Z_{2} \times Z_{2}^{\prime}$.

${ }^{2}$ A multicomponent DM system has been considered recently in Refs. [18 21]; see also the references therein.
} 
TABLE I: The matter content of the model and the corresponding quantum numbers. $Z_{2} \times Z_{2}^{\prime}$ is the unbroken discrete symmetry, while the lepton number $\mathrm{L}$ is softly broken by the $\phi$ mass. $D_{2 N}(N=2,3, \ldots)$ is the dihedral group of order $2 N$.

\begin{tabular}{|c|c|c|c|c|c|c||c|}
\hline Field & Statistics & $S U(2)_{L}$ & $U(1)_{Y}$ & $\mathrm{~L}$ & $Z_{2}$ & $Z_{2}^{\prime}$ & $D_{2 N}$ \\
\hline$L=\left(\nu_{L}, l_{L}\right)$ & $\mathrm{F}$ & 2 & $-1 / 2$ & 1 & + & + & $\mathbf{1}$ \\
\hline$l_{R}^{c}$ & $\mathrm{~F}$ & 1 & 1 & -1 & + & + & $\mathbf{1}$ \\
\hline$N_{R}^{c}$ & $\mathrm{~F}$ & 1 & 0 & 0 & - & + & $\mathbf{1}^{\prime \prime}$ \\
\hline$H=\left(H^{+}, H^{0}\right)$ & $\mathrm{B}$ & 2 & $1 / 2$ & 0 & + & + & $\mathbf{1}$ \\
\hline$\eta=\left(\eta^{+}, \eta^{0}\right)$ & $\mathrm{B}$ & 2 & $1 / 2$ & -1 & - & + & $\mathbf{1}^{\prime \prime}$ \\
\hline$\chi$ & $\mathrm{B}$ & 1 & 0 & 0 & + & - & $\mathbf{1}^{\prime}$ \\
\hline$\phi$ & $\mathrm{B}$ & 1 & 0 & 1 & - & - & $\mathbf{1}^{\prime \prime \prime}$ \\
\hline
\end{tabular}

boson $h$. The results of the LHC indicate a slight excess of $h \rightarrow \gamma \gamma$ [25 27], which in fact could be explained by an additional inert doublet circulating in one loop 28 31]. There is yet another excess of $\gamma$ at the Fermi Large Area Telescope (LAT) [32 35]. There are analyses [36 41] that indicate a monochromatic $\gamma$-ray line of $135 \mathrm{GeV}$ in the Fermi data. It has been reported [42] that a two-component DM system consisting of an inert doublet scalar and a scalar can explain the monochromatic $\gamma$-ray line at the Fermi LAT. Several models with a two-component DM have also been considered in Refs. [43, 44] to explain the monochromatic Fermi LAT $\gamma$-ray line. Therefore it is natural to wonder whether our two-loop radiative seesaw model can explain the $\gamma$ excess in the Higgs boson decay as well as in the Fermi data ${ }^{3}$. We find that this is in fact possible if we accept that certain scalar couplings are large at the border of perturbation theory, where to suppress sufficiently the continuum $\gamma$ 's and the production of antiprotons, we employ a mechanism of temperaturedependent annihilation cross section [50, 51].

\section{THE MODEL}

The matter content of the model is shown in Table I. The new fields are (in addition to the right-handed neutrino $\left.N_{R}^{c}\right)$ the $S U(2)_{L}$ doublet scalar $\eta(\mathrm{L}=-1)$ and singlet scalars $\chi$ $(\mathrm{L}=0)$ and $\phi(\mathrm{L}=1)$, where $\mathrm{L}$ is the lepton number. Note that the $\mathrm{L}$ of $N_{R}^{c}$ is zero and that four different representations of $Z_{2} \times Z_{2}^{\prime}$ are exactly the singlets of the dihedral group of order $2 N, D_{2 N}(N=2,3, \ldots)$. The $Z_{2} \times Z_{2}^{\prime} \times \mathrm{L}$-invariant (or $D_{2 N} \times \mathrm{L}$-invariant) Yukawa

\footnotetext{
3 Recently, it has been argued that the monochromatic $\gamma$-ray line can be explained basically by the same one-loop contribution as for $h \rightarrow \gamma \gamma$ [42, 45, 49].
} 
couplings of the lepton sector can be described by

$$
\mathcal{L}_{Y}=Y_{i j}^{e} H^{\dagger} L_{i} l_{R j}^{c}+Y_{i k}^{\nu} L_{i} \epsilon \eta N_{R k}^{c}+\text { h.c. },
$$

with the Majorana mass term of the right-handed neutrinos $N_{R k}^{c}(k=1,2,3)$

$$
\mathcal{L}_{\mathrm{Maj}}=-\frac{1}{2}\left[M_{k} N_{R k}^{c} N_{R k}^{c}+\text { h.c. }\right] .
$$

The most general form of the $Z_{2} \times Z_{2}^{\prime} \times$ L-invariant scalar potential is given by

$$
\begin{aligned}
V_{\lambda}= & \lambda_{1}\left(H^{\dagger} H\right)^{2}+\lambda_{2}\left(\eta^{\dagger} \eta\right)^{2}+\lambda_{3}\left(H^{\dagger} H\right)\left(\eta^{\dagger} \eta\right)+\lambda_{4}\left(H^{\dagger} \eta\right)\left(\eta^{\dagger} H\right) \\
& +\gamma_{1} \chi^{4}+\gamma_{2}\left(H^{\dagger} H\right) \chi^{2}+\gamma_{3}\left(\eta^{\dagger} \eta\right) \chi^{2}+\gamma_{4}|\phi|^{4}+\gamma_{5}\left(H^{\dagger} H\right)|\phi|^{2} \\
& +\gamma_{6}\left(\eta^{\dagger} \eta\right)|\phi|^{2}+\gamma_{7} \chi^{2}|\phi|^{2}+\frac{\kappa}{2}\left[\left(H^{\dagger} \eta\right) \chi \phi+\text { h.c. }\right] .
\end{aligned}
$$

Note that the " $\lambda_{5}$ term", $(1 / 2) \lambda_{5}\left(H^{\dagger} \eta\right)^{2}$, is forbidden by L. The $Z_{2} \times Z_{2}^{\prime}$-invariant mass term is

$$
V_{m}=m_{1}^{2} H^{\dagger} H+m_{2}^{2} \eta^{\dagger} \eta+\frac{1}{2} m_{3}^{2} \chi^{2}+m_{4}^{2}|\phi|^{2}+\frac{1}{2} m_{5}^{2}\left[\phi^{2}+\left(\phi^{*}\right)^{2}\right],
$$

where the last term in Eq. (4) breaks L softly. This is the only $Z_{2} \times Z_{2}^{\prime}$-invariant mass term which can break L softly. In the absence of this term, there will be no neutrino mass. The charged CP-even and CP-odd scalars are defined as

$$
H=\left(\begin{array}{c}
H^{+} \\
\left(v_{h}+h+i G\right) / \sqrt{2}
\end{array}\right), \eta=\left(\begin{array}{c}
\eta^{+} \\
\left(\eta_{R}^{0}+i \eta_{I}^{0}\right) / \sqrt{2}
\end{array}\right), \phi=\left(\phi_{R}+i \phi_{I}\right) / \sqrt{2} .
$$

The tree-level masses of the scalars are given by

$$
\begin{aligned}
m_{h}^{2} & =2 \lambda_{1} v_{h}^{2}, m_{\eta^{ \pm}}^{2}=m_{2}^{2}+\frac{1}{2} \lambda_{3} v_{h}^{2}, m_{\eta_{R}^{0}}^{2}=m_{\eta_{I}^{0}}^{2}=m_{2}^{2}+\frac{1}{2}\left(\lambda_{3}+\lambda_{4}\right) v_{h}^{2}, \\
m_{\phi_{R}}^{2} & =m_{4}^{2}+m_{5}^{2}+\gamma_{5} v_{h}^{2}, m_{\phi_{I}}^{2}=m_{4}^{2}-m_{5}^{2}+\gamma_{5} v_{h}^{2}, m_{\chi}^{2}=m_{3}^{2}+\gamma_{2} v_{h}^{2} .
\end{aligned}
$$

A supersymmetric $S U(5)$ UV completion of the model in the spirit of Ref. [52] is possible, where the scalar masses are protected from a large correction coming from the quadratic divergence. In this case, the soft breaking of the lepton number will appear as a softsupersymmetric-breaking B term. Another way to get rid of the quadratic divergence without introducing supersymmetry is given in Refs. [53, 54], where classical conformal symmetry is used. In this treatment the quadratic divergence in the scalar masses does nothing wrong, so there is no need to protect them.

\section{A. Stability of the vacuum}

The potential $V_{\lambda}$ is bounded below if

$$
\lambda_{1}, \lambda_{2}, \gamma_{1}, \gamma_{4}>0
$$




$$
\begin{aligned}
& \lambda_{3}>-\frac{2}{3} \sqrt{\lambda_{1} \lambda_{2}}, \quad \lambda_{3}+\lambda_{4}>-\frac{2}{3} \sqrt{\lambda_{1} \lambda_{2}} \\
& \gamma_{2}>-\frac{2}{3} \sqrt{\lambda_{1} \gamma_{1}}, \quad \gamma_{5}>-\frac{2}{3} \sqrt{\lambda_{1} \gamma_{4}}, \quad \gamma_{3}>-\frac{2}{3} \sqrt{\lambda_{2} \gamma_{1}} \\
& \gamma_{6}>-\frac{2}{3} \sqrt{\lambda_{2} \gamma_{4}}, \quad \gamma_{7}>-\frac{2}{3} \sqrt{\gamma_{1} \gamma_{4}} \\
& \lambda_{1}+\lambda_{2}+\gamma_{1}+\gamma_{4}-\frac{2}{3}\left(\sqrt{\lambda_{1} \lambda_{2}}+\sqrt{\lambda_{1} \gamma_{1}}+\sqrt{\lambda_{1} \gamma_{4}}\right. \\
& \left.+\sqrt{\lambda_{2} \gamma_{1}}+\sqrt{\lambda_{2} \gamma_{4}}+\sqrt{\gamma_{1} \gamma_{4}}\right)>|\kappa|
\end{aligned}
$$

are satisfied. The minimum of $V_{\lambda}$ is zero if the inequalities above are satisfied. The discrete symmetry $Z_{2} \times Z_{2}^{\prime}$ (or $D_{2 N}$ ) is unbroken if, in addition to Eqs. (7)-(11), the inequalities $m_{2}^{2}, m_{3}^{2}, m_{4}^{2}$ and $m_{4}^{2}-\left|m_{5}\right|^{2}>0$ are satisfied.

The inequalities of Eqs. (7)-(11) are sufficient conditions, but not necessary ones. If we assume that Eq. (7) and $\gamma_{2}, \gamma_{3}, \gamma_{5}, \gamma_{6}, \gamma_{7}>0$ are satisfied, for instance, the inequalities of Eq. (8) are relaxed to

$$
\lambda_{3}, \lambda_{3}+\lambda_{4}>-2 \sqrt{\lambda_{1} \lambda_{2}}
$$

and Eq. (11) is relaxed to

$$
\lambda_{1}+\lambda_{2}+\gamma_{1}+\sum_{i=2}^{7} \gamma_{i}-2 \sqrt{\lambda_{1} \lambda_{2}}>|\kappa| .
$$

Since $m_{h}=125 \mathrm{GeV}$ and $v_{h}=246 \mathrm{GeV}$, the Higgs coupling $\lambda_{1}$ is fixed at 0.129 . Then Eq. (12) implies that

$$
\lambda_{3}, \lambda_{3}+\lambda_{4}>-2.5 \sqrt{\lambda_{2} / 4 \pi}
$$

\section{B. Neutrino mass}

The neutrino masses can be generated at the two-loop level as shown in Fig. 1. The mechanism of the radiative generation is the following: Because of the soft breaking of the dimension-two operator $\phi^{2}$, the propagator between $\phi$ and $\phi$ can exist. This can generate a $\eta^{0} \eta^{0}$ mass term. In the one-loop radiative seesaw model of Ref. [8] this mass is generated at the tree level through the " $\lambda_{5}$ " coupling. So the effective $\lambda_{5}^{\text {eff }}$ is

$$
\lambda_{5}^{\mathrm{eff}}=-\frac{\kappa^{2}}{64 \pi^{2}}\left[\frac{m_{\phi_{I}}^{2}}{m_{\phi_{I}}^{2}-m_{\chi}^{2}} \ln \frac{m_{\phi_{I}}^{2}}{m_{\chi}^{2}}-\frac{m_{\phi_{R}}^{2}}{m_{\phi_{R}}^{2}-m_{\chi}^{2}} \ln \frac{m_{\phi_{R}}^{2}}{m_{\chi}^{2}}\right] .
$$

Therefore, $\lambda_{5}^{\text {eff }}$ cannot be large within the framework of perturbation theory $\left(\lambda_{5}^{\text {eff }} \lesssim O\left(10^{-2}\right)\right)$. For a set of parameter values

$$
m_{\chi}=135 \mathrm{GeV}, m_{\phi_{R}}=300 \mathrm{GeV}, m_{\phi_{I}}=700 \mathrm{GeV}, \kappa=3
$$




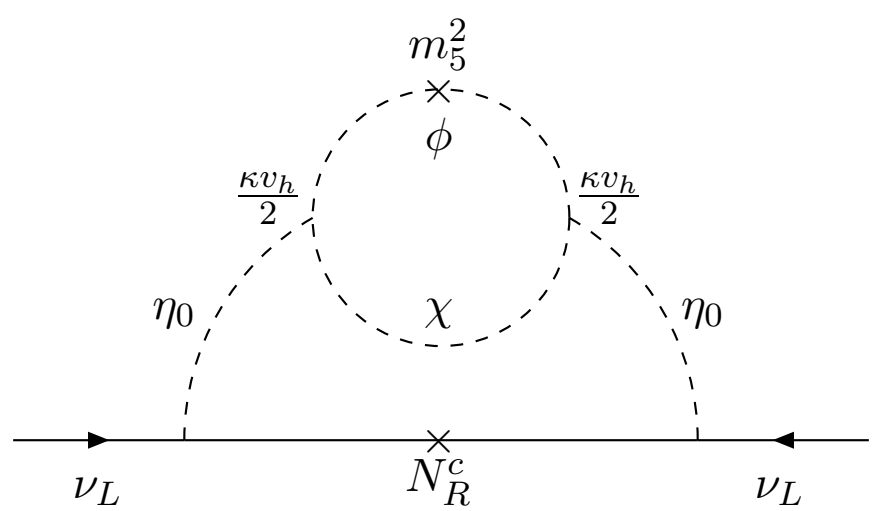

FIG. 1: Two-loop radiative neutrino mass.

for instance, we obtain $\lambda_{5}^{\text {eff }}=-0.02$. The two-loop neutrino mass matrix is calculated to be

$$
\begin{aligned}
\left(\mathcal{M}_{\nu}\right)_{i j}= & \left(\frac{1}{16 \pi^{2}}\right)^{2} \frac{\kappa^{2} v_{h}^{2}}{8} \sum_{k} Y_{i k}^{\nu} Y_{j k}^{\nu} M_{k} \int_{0}^{\infty} d x\left\{B_{0}\left(-x, m_{\chi}, m_{\phi_{R}}\right)-B_{0}\left(-x, m_{\chi}, m_{\phi_{I}}\right)\right\} \\
& \times \frac{x}{\left(x+m_{\eta}^{2}\right)^{2}\left(x+M_{k}^{2}\right)} \text { for } m_{\eta}=m_{\eta_{R}^{0}} \simeq m_{\eta_{I}^{0}}
\end{aligned}
$$

where the function $B_{0}$ is the Passarino-Veltman function [55]

$$
\frac{i}{16 \pi^{2}} B_{0}\left(p^{2}, m_{1}, m_{2}\right)=\int \frac{d^{D} k}{(2 \pi)^{D}} \frac{1}{\left(k^{2}-m_{1}^{2}+i \epsilon\right)\left((k+p)^{2}-m_{2}^{2}+i \epsilon\right)} .
$$

For the set of parameters given in Eq. (16) with $M_{k}=1 \mathrm{TeV}, m_{\eta}=150 \mathrm{GeV}$ and $v_{h}=246$ $\mathrm{GeV}$ we obtain $\left(\mathcal{M}_{\nu}\right)_{i j} \simeq-0.7 \times 10^{7} \sum_{k} Y_{i k}^{\nu} Y_{j k}^{\nu} \mathrm{eV}$, and so the neutrino mass will be $O\left(10^{-1}\right)$ $\mathrm{eV}$ if $\sum_{k} Y_{i k}^{\nu} Y_{j k}^{\nu}=10^{-8}$. If $m_{\eta} \ll M_{k}$, Eq. (17) can be estimated as ${ }^{4}$

$$
\left(\mathcal{M}_{\nu}\right)_{i j} \sim-\lambda_{5}^{\mathrm{eff}} v_{h}^{2} \sum_{k} \frac{Y_{i k}^{\nu} Y_{j k}^{\nu}}{16 \pi^{2} M_{k}}\left(\ln \left(\frac{m_{\eta_{R}^{0}}}{M_{k}}\right)^{2}+1\right) \text { for } m_{\eta} \ll M_{k} .
$$

Therefore, the scale of the light neutrino mass will be

$$
\frac{\kappa^{2}}{64 \pi^{2}} \frac{1}{16 \pi^{2}} \frac{m_{D}^{2}}{M} \sim\left(\frac{\kappa}{0.1}\right)^{2} 10^{-7} \times \frac{m_{D}^{2}}{M}
$$

where $m_{D}^{2} / M$ is the scale in the case of the tree-level type-I seesaw. This means that we can scale down the mass of the right-handed neutrino by several orders of magnitude. So, the right-handed neutrino masses of $\mathrm{TeV}$ or less are naturally expected in this model.

\footnotetext{
${ }^{4}$ There is $O(1)$ correction to the approximate formula Eq. (19), which we have checked numerically.
} 


\section{Constraints}

1: $\mu \rightarrow e \gamma$

The constraint coming from $\mu \rightarrow e \gamma$ is given by [56]

$$
\begin{aligned}
& B(\mu \rightarrow e \gamma)=\frac{3 \alpha}{64 \pi\left(G_{F} m_{\eta^{ \pm}}^{2}\right)^{2}}\left|\sum_{k} Y_{\mu k}^{\nu} Y_{e k}^{\nu} F_{2}\left(\frac{M_{k}^{2}}{m_{\eta^{ \pm}}^{2}}\right)\right|^{2} \lesssim 2.4 \times 10^{-12}, \\
& F_{2}(x)=\frac{1}{6(1-x)^{4}}\left(1-6 x+3 x^{2}+2 x^{3}-6 x^{2} \ln x\right)
\end{aligned}
$$

where the upper bound is taken from Ref. [57]. A similar, but slightly weaker bound for $\tau \rightarrow \mu(e) \gamma$ given in Ref. [57] has to be satisfied, too. Since $F_{2}(x) \sim 1 / 3 x$ for $x \gg 1$, while $1 / 12<F_{2}(x)<1 / 6$ for $0<x<1$, the constraint can be readily satisfied if $M_{k} \ll m_{\eta^{ \pm}}$ or $M_{k} \gg m_{\eta^{ \pm}}$. If we assume that $M_{1}=M_{2}=M_{3}=1 \mathrm{TeV} \gg m_{\eta^{ \pm}}$in Eq. (21), the constraint of Eq. (21) becomes $B(\mu \rightarrow e \gamma) \simeq 10^{-7} \times\left|\sum_{k} Y_{\mu k}^{\nu} Y_{e k}^{\nu}\right|^{2} \lesssim 2.4 \times 10^{-12}$. Therefore, $\left|Y_{e k}^{\nu} Y_{\mu k}^{\nu}\right|^{2} \lesssim O\left(10^{-5}\right)$ can satisfy the constraint.

2: $\underline{g_{\mu}-2}$

The extra contribution to the anomalous magnetic moment of the muon, $a_{\mu}=\left(g_{\mu}-2\right) / 2$, is given by [56]

$$
\delta a_{\mu}=\frac{m_{\mu}^{2}}{16 \pi^{2} m_{\eta \pm}^{2}} \sum_{k} Y_{\mu k}^{\nu} Y_{\mu k}^{\nu} F_{2}\left(\frac{M_{k}^{2}}{m_{\eta^{ \pm}}^{2}}\right) .
$$

If we assume that $\left|\sum_{k} Y_{\mu k}^{\nu} Y_{\mu k}^{\nu} F_{2}\left(\frac{M_{k}^{2}}{m_{\eta^{ \pm}}^{2}}\right)\right| \simeq\left|\sum_{k} Y_{\mu k}^{\nu} Y_{e k}^{\nu} F_{2}\left(\frac{M_{k}^{2}}{m_{\eta^{ \pm}}^{2}}\right)\right|$, then we obtain

$$
\left|\delta a_{\mu}\right| \simeq 1.4 \times 10^{-7} B(\mu \rightarrow e \gamma)^{1 / 2},
$$

where the upper bound on $\left|\delta a_{\mu}\right|$ is given by $3.4 \times 10^{-11}\left[58\right.$ ]. So, the constraint from $a_{\mu}$ has no significant influence, if the constraint of Eq. (21) is satisfied.

3: Electroweak precision

The electroweak precision measurement requires [58, 59]

$$
\Delta T \simeq 1.08\left(\frac{m_{\eta^{ \pm}}-m_{\eta_{R}^{0}}}{v}\right)\left(\frac{m_{\eta^{ \pm}}-m_{\eta_{I}^{0}}}{v}\right)=0.07 \pm 0.08
$$

for $m_{h}=115.5-127 \mathrm{GeV}$. Therefore, $\left|m_{\eta^{ \pm}}-m_{\eta_{R}^{0}}\right|,\left|m_{\eta^{ \pm}}-m_{\eta_{I}^{0}}\right| \lesssim 90 \mathrm{GeV}$ is sufficient to meet the requirement.

\section{DM AND RESTRICTING THE PARAMETER SPACE}

A. $h \rightarrow \gamma \gamma$

Because of the coupling $\mathcal{L}_{H^{\dagger} H \eta^{\dagger} \eta}=-\lambda_{3}\left(H^{\dagger} H\right)\left(\eta^{\dagger} \eta\right)=-\lambda_{3} v_{h} h \eta^{+} \eta^{-}+\cdots$, there are additional diagrams that contribute to the one-loop decay $h \rightarrow \gamma \gamma$, which are shown in 


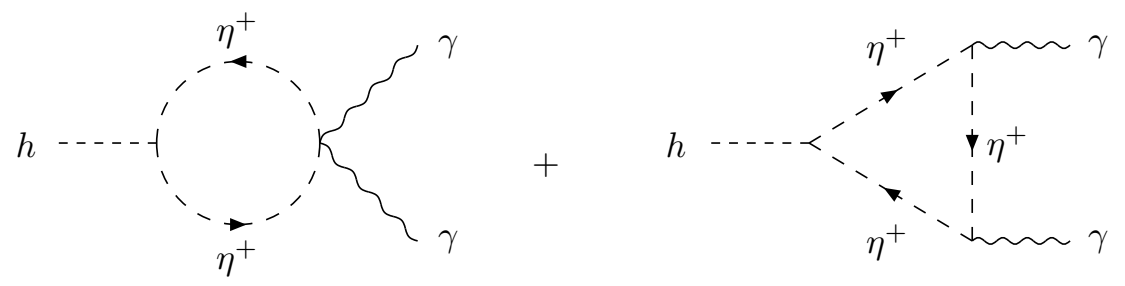

FIG. 2: One-loop diagrams for $h \rightarrow \gamma \gamma$.

Fig. 2. Therefore, the decay width for two $\gamma^{\prime}$ 's can be increased [28 31]:

$$
\begin{aligned}
\frac{\Gamma(\gamma \gamma)}{\Gamma^{\operatorname{SM}}(\gamma \gamma)} & =\left[\frac{3(3 / 2)^{2} F_{1 / 2}\left(\tau_{t}\right)+F_{1}\left(\tau_{W}\right)+2 \lambda_{3}\left(m_{W}^{2} / g^{2} m_{\eta^{ \pm}}^{2}\right) F_{0}\left(\tau_{\eta^{ \pm}}\right)}{3(3 / 2)^{2} F_{1 / 2}\left(\tau_{t}\right)+F_{1}\left(\tau_{W}\right)}\right]^{2} \\
\tau_{t} & =4 m_{t}^{2} / m_{h}^{2}, \tau_{W}=4 m_{W}^{2} / m_{h}^{2}, \tau_{\eta^{ \pm}}=4 m_{\eta^{ \pm}}^{2} / m_{h}^{2} \\
F_{1 / 2}(\tau) & =2+3 \tau+3 \tau(2-\tau) \arcsin ^{2}(1 / \sqrt{\tau}) \\
F_{1}(\tau) & =-2 \tau\left[1+(1-\tau) \arcsin ^{2}(1 / \sqrt{\tau})\right] \\
F_{0}(\tau) & =\tau\left[1-\tau \arcsin ^{2}(1 / \sqrt{\tau})\right]
\end{aligned}
$$

where $\arcsin ^{2}(1 / \sqrt{\tau})$ should be replaced by $(-1 / 4)\left[\ln \frac{1+\sqrt{1+\tau}}{1-\sqrt{1-\tau}}-i \pi\right]^{2}$ for $\tau<1$. We obtain

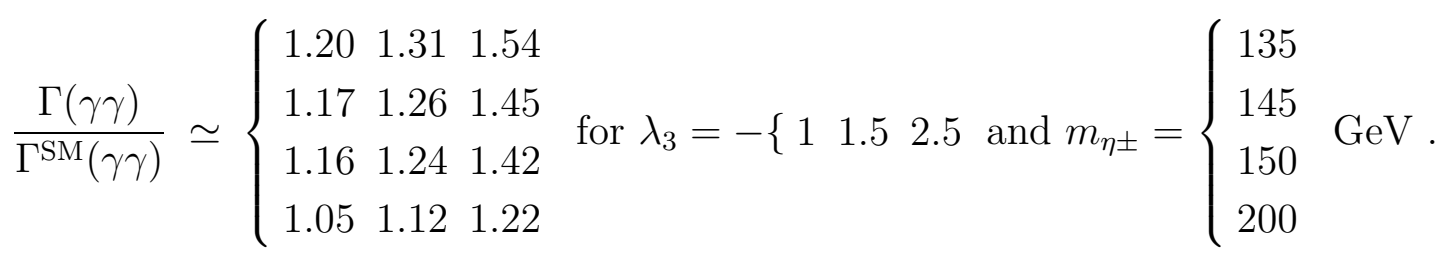

So, if the charged inert scalar $\eta^{+}$is relatively light and $\lambda_{3}$ is negative and large, the observed excess $1.6 \pm 0.4$ in the CMS experiment [26] can be explained. (The best-fit signal strength for this mode in the ATLAS experiment [25, 27] is $\hat{\mu}=1.9 \pm 0.5$.) In Fig. 3 we show the area in the $m_{\eta^{ \pm}}-\lambda_{3}$ plane in which $\Gamma(\gamma \gamma) / \Gamma^{\mathrm{SM}}(\gamma \gamma)=1.6 \pm 0.4$ can be obtained ${ }^{5}$. As we can see from Eq. (8) , a large negative $\lambda_{3}$ may endanger the vacuum stability, because $\lambda_{1}$ is fixed at 0.129. Therefore, we assume that all the quartic scalar couplings except $\lambda_{3}$ and $\lambda_{4}$ are positive and use the second set of the inequality conditions in Eqs. (12) and (13). Equation (14) means that $\lambda_{3} \gtrsim-2.5$ if $\lambda_{2}$ is at the border of perturbation theory. Thus, the Higgs boson decay mode $h \rightarrow \gamma \gamma$ prefers the parameter space:

${ }^{5}$ The upper bound $m_{\eta^{ \pm}}<135 \mathrm{GeV}$ given in Ref. [31] is obtained from $\Gamma(\gamma \gamma) / \Gamma^{\mathrm{SM}}(\gamma \gamma)>1.3$ and $\lambda_{3} / 4 \pi>$ $-1.46 / 4 \pi \simeq-0.116$, which is consistent with Eq. (26). Note that we use $2.0 \geq \Gamma(\gamma \gamma) / \Gamma^{\mathrm{SM}}(\gamma \gamma) \geq 1.2$ for Fig. 3 along with the stability constraint [Eq. (14)]. 


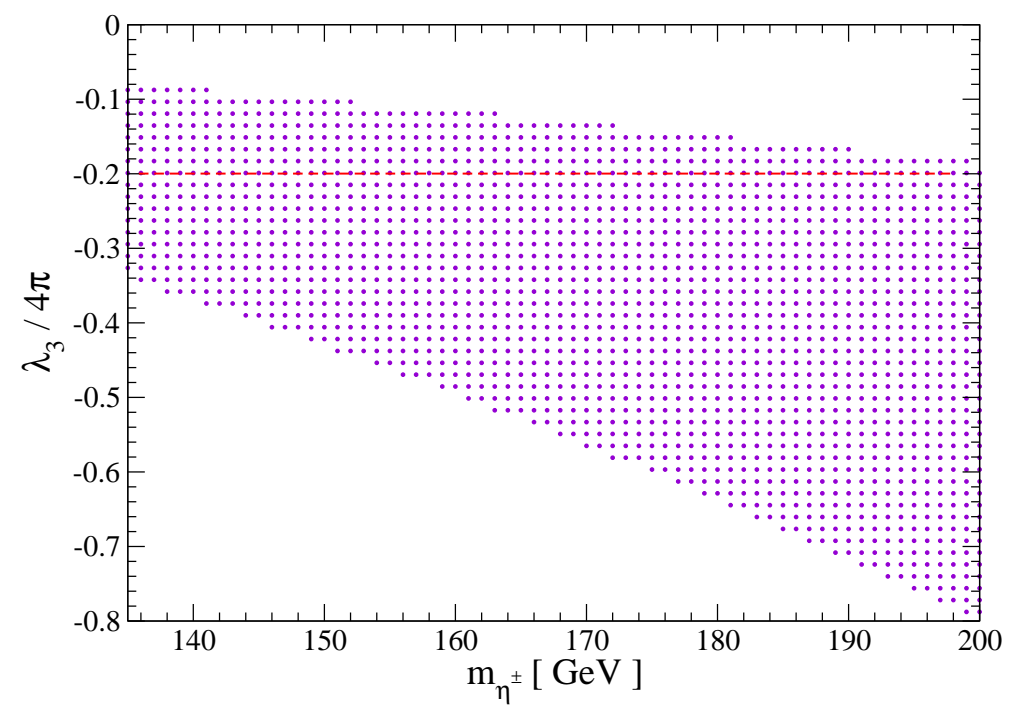

FIG. 3: The area with $\Gamma / \Gamma^{\mathrm{SM}}=1.6 \pm 0.4$ in the $m_{\eta^{ \pm}}-\lambda_{3}$ plane. The horizontal red line is the stability bound in given in Eq. (14).

1. All the quartic scalar couplings except $\lambda_{3}$ and $\lambda_{4}$ are positive, and $\lambda_{2}$ is large.

2. The constraints

$$
-0.2 \lesssim \lambda_{3} / 4 \pi \lesssim-0.08 \text { and } m_{\eta^{ \pm}} \lesssim 200 \mathrm{GeV}
$$

have to be satisfied.

\section{B. Direct detection of DM}

As we can see from Table I, either $N_{R}^{c}$ or $\eta$ can be a DM candidate. Here we assume that $\eta_{R}^{0}$, the CP-even component of $\eta$, is a DM particle and assume that $M_{k} \gg m_{\eta_{R}^{0}}, m_{\eta_{I}^{0}}, m_{\eta^{ \pm}}$ to satisfy the $\mu \rightarrow e \gamma$ constraint [Eq. (21)]. The model can have three stable DM particles in principle, but to simplify the situation we assume a two-component DM system. Another one is either $\chi$ or $\phi$. As we see from the potential [Eq. (3)], there is no significant difference between $\chi$ and $\phi$ as DM. So we assume here that $\chi$ is the second DM particle. [59]:

The spin-independent elastic cross sections off the nucleon, $\sigma(\chi)$ and $\sigma\left(\eta_{R}^{0}\right)$, are given by

$$
\sigma(\chi)=\frac{1}{\pi}\left(\frac{\gamma_{2} \hat{f} m_{N}}{m_{\chi} m_{h}^{2}}\right)^{2}\left(\frac{m_{N} m_{\chi}}{m_{N}+m_{\chi}}\right)^{2}, \sigma\left(\eta_{R}^{0}\right)=\frac{1}{\pi}\left(\frac{\lambda_{L} \hat{f} m_{N} / 2}{m_{\eta_{R}^{0}} m_{h}^{2}}\right)^{2}\left(\frac{m_{N} m_{\eta_{R}^{0}}}{m_{N}+m_{\eta_{R}^{0}}}\right)^{2},
$$

where $\lambda_{L}=\lambda_{3}+\lambda_{4}, m_{N}$ is the nucleon mass, and $\hat{f} \sim 0.3$ stems from the nucleonic matrix element [60]. The cross sections have to satisfy

$$
\left(\frac{\sigma(\chi)}{\sigma_{\mathrm{UB}}\left(m_{\chi}\right)}\right)\left(\frac{\Omega_{\chi} h^{2}}{\Omega_{T} h^{2}}\right)+\left(\frac{\sigma\left(\eta_{R}^{0}\right)}{\sigma_{\mathrm{UB}}\left(m_{\eta_{R}^{0}}\right)}\right)\left(\frac{\Omega_{\eta} h^{2}}{\Omega_{T} h^{2}}\right) \lesssim 1
$$



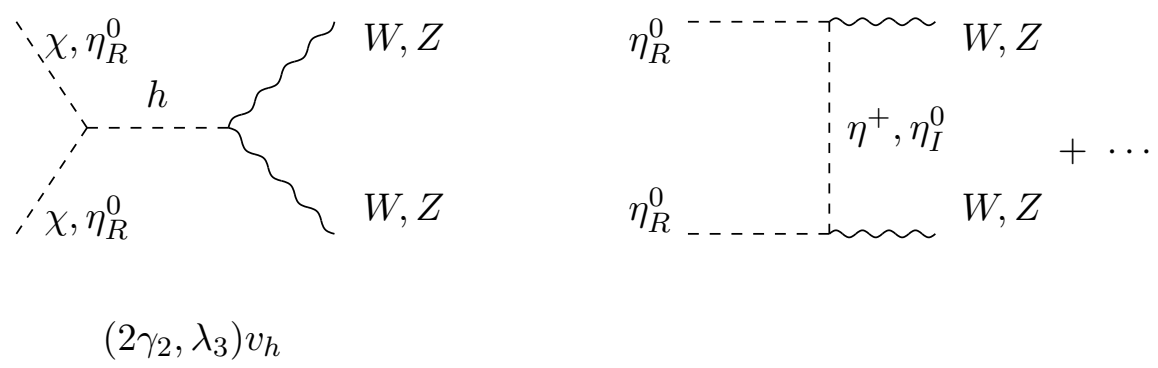

FIG. 4: Tree-level DM annihilations into $W^{+} W^{-}$and $Z Z$.

where $\Omega_{T} h^{2}=0.116$ [61], and $\sigma_{\mathrm{UB}}(m) \simeq 3 \times 10^{-45} \mathrm{~cm}^{2}[62]$ is the XENON100 limit for the DM mass of $135 \mathrm{GeV}$. So, we find

$$
\begin{aligned}
\left|\gamma_{2}\right| & \lesssim 0.035(0.003) \text { if } \Omega_{\chi} h^{2} \simeq \Omega_{T} h^{2}=0.116 \text { and } m_{\chi}=135 \mathrm{GeV} \\
\left|\lambda_{3}+\lambda_{4}\right| & \lesssim 0.069(0.006) \text { if } \Omega_{\eta} h^{2} \simeq \Omega_{T} h^{2}=0.116 \text { and } m_{\eta_{R}^{0}}=135 \mathrm{GeV}
\end{aligned}
$$

where we would obtain the numbers in the parentheses when the XENON1T sensitivity $\sigma \sim 2 \times 10^{-47} \mathrm{~cm}^{2}$ [63] has been reached. Because of Eq. (27), we have to assume a large negative $\lambda_{3}$ and, because of $m_{\eta^{ \pm}}^{2}-m_{\eta_{R}^{0}}^{2}=-\lambda_{4} v_{h}^{2} / 2, \lambda_{4}$ has to be negative, too, to ensure $m_{\eta^{ \pm}}>m_{\eta_{R}^{0}}$. If $\lambda_{4}$ is negative and in addition $\lambda_{3}$ is large and negative, the inequality of Eq. (12) may be violated, unless $\lambda_{4}$ is small and negative. This means $\lambda_{L} \simeq \lambda_{3}$, implying that Eq. (31) cannot be satisfied. Therefore, the relic density of $\eta_{R}^{0}$ has to be small to satisfy the constraint of Eq. (29). This is welcome, because the annihilation cross section of $\eta_{R}^{0}$ is large in general due to the gauge interactions shown in Fig. 4 [22 24]. Thus, the parameter space is further constrained:

1. $\lambda_{4}$ has to be negative and small to ensure $m_{\eta^{ \pm}}>m_{\eta_{R}^{0}}$ and to satisfy the constraint of Eq. (14).

2. Since $\lambda_{3}$ is assumed to be large and negative [ see Eq. (27) ], the constraint of Eq. (29) can be satisfied only if $\Omega_{\eta} / \Omega_{T} \ll 1$.

3. To satisfy the XENON100 (1T) constraint, we have to impose $\left|\gamma_{2}\right| \lesssim 0.035(0.003)$ for $m_{\chi}=135 \mathrm{GeV}$.

\section{Relic densities of DM}

Since our parameter space has already been constrained to a certain amount, we next calculate the relic density of DM, $\Omega_{T}=\Omega_{\chi}+\Omega_{\eta}$. To simplify the situation we have been assuming throughout that $\eta_{R}^{0}$ and $\chi$ are DM particles. In this two-component DM system 
there are three different thermally averaged cross sections

$$
\left\langle\sigma\left(\eta_{R}^{0} \eta_{R}^{0} ; \mathrm{SM}\right) v\right\rangle,\langle\sigma(\chi \chi ; \mathrm{SM}) v\rangle,\left\langle\sigma\left(\eta_{R}^{0} \eta_{R}^{0} ; \chi \chi\right) v\right\rangle
$$

that are relevant for calculating the DM relic density, where SM stands for the SM particles ${ }^{6}$. No semiannihilation $\eta_{R}^{0} \chi \rightarrow \phi_{R(I)} \mathrm{SM}$ is allowed if $m_{\phi_{R(I)}}>m_{\eta_{R}^{0}}+m_{\chi}$. Then the evolution equation for $Y$, the number density over the entropy density, can be written as [18 21]

$$
\begin{aligned}
\frac{d Y_{\eta_{R}^{0}}}{d x}= & -0.264 g_{*}^{1 / 2}\left[\frac{\mu M_{\mathrm{PL}}}{x^{2}}\right]\left\{\left\langle\sigma\left(\eta_{R}^{0} \eta_{R}^{0} ; \mathrm{SM}\right) v\right\rangle\left(Y_{\eta_{R}^{0}} Y_{\eta_{R}^{0}}-\bar{Y}_{\eta_{R}^{0}} \bar{Y}_{\eta_{R}^{0}}\right)\right. \\
& \left.+\left\langle\sigma\left(\eta_{R}^{0} \eta_{R}^{0} ; \chi \chi\right) v\right\rangle\left(Y_{\eta_{R}^{0}} Y_{\eta_{R}^{0}}-\frac{Y_{\chi} Y_{\chi}}{\bar{Y}_{\chi} \bar{Y}_{\chi}} \bar{Y}_{\eta_{R}^{0}}\right)\right\}, \\
\frac{d Y_{\chi}}{d x}= & -0.264 g_{*}^{1 / 2}\left[\frac{\mu M_{\mathrm{PL}}}{x^{2}}\right]\left\{\langle\sigma(\chi \chi ; \mathrm{SM}) v\rangle\left(Y_{\chi} Y_{\chi}-\bar{Y}_{\chi} \bar{Y}_{\chi}\right)\right. \\
& \left.-\left\langle\sigma\left(\eta_{R}^{0} \eta_{R}^{0} ; \chi \chi\right) v\right\rangle\left(Y_{\eta_{R}^{0}} Y_{\eta_{R}^{0}}-\frac{Y_{\chi} Y_{\chi}}{\bar{Y}_{\chi} \bar{Y}_{\chi}} \bar{Y}_{\eta_{R}^{0}} \bar{Y}_{\eta_{R}^{0}}\right)\right\},
\end{aligned}
$$

where $\bar{Y}$ is $Y$ in equilibrium, $x=\mu / T, 1 / \mu=1 / m_{\eta_{R}^{0}}+1 / m_{\chi}$, and $T, M_{\mathrm{PL}}$ and $g_{*}=90$ are the temperature, the Planck mass and the total number of effective degrees of freedom, respectively.

Before we solve the evolution equations numerically, we consider what we would expect. As noticed, the relic density of $\eta_{R}^{0}$ will be very small because of large $\lambda_{3}$ and gauge interactions (i.e. large $\left.\left\langle\sigma\left(\eta_{R}^{0} \eta_{R}^{0} ; \mathrm{SM}\right) v\right\rangle\right)$, while the annihilation cross section of $\chi$ into the SM particles is suppressed because of Eq. (30) (i.e. small $\langle\sigma(\chi \chi ; \mathrm{SM}) v\rangle)$. That is, the DM conversion cross section $\left\langle\sigma\left(\eta_{R}^{0} \eta_{R}^{0} ; \chi \chi\right) v\right\rangle$ and the mass difference $\Delta m_{\eta \chi}=m_{\eta_{R}^{0}}-m_{\chi}$ will play an important role. Note that the smaller $\Delta m_{\eta \chi}$ is, the larger is the effect of the DM conversion on $\Omega_{\chi}$. To see this more explicitly, we assume that $\eta_{R}^{0}$ annihilates very fast so that before and at the decoupling of $\chi$ the $\eta_{R}^{0} \mathrm{DM}$ is in thermal equilibrium. Then the expression in \{\} in the rhs of Eq. (34) can be written as

$$
\left[\langle\sigma(\chi \chi ; \mathrm{SM}) v\rangle+\left\langle\sigma\left(\eta_{R}^{0} \eta_{R}^{0} ; \chi \chi\right) v\right\rangle \frac{m_{\eta_{R}^{0}}^{3}}{m_{\chi}^{3}} \exp \left(2 x \frac{m_{\chi}^{2}-m_{\eta_{R}^{0}}^{2}}{m_{\chi} m_{\eta_{R}^{0}}}\right)\right]\left(Y_{\chi} Y_{\chi}-\bar{Y}_{\chi} \bar{Y}_{\chi}\right)
$$

which also appears in the coannihilation of DM with an unstable particle [50] ${ }^{7}$. If $m_{\chi}^{2}-$ $m_{\eta_{R}^{0}}^{2}<0$, the effective annihilation cross section of $\chi$ is small at low temperature (large $x$ ), while it is large at high temperature ( $\operatorname{small} x)$. Because of the nontrivial interplay between $\gamma_{2}$ and $\Delta m_{\eta \chi}$, it may be possible to obtain a correct relic density $\Omega_{T} h^{2}=0.1157 \pm 0.0023$

\footnotetext{
6 These thermally averaged cross sections are tree-level ones and do not include those into two $\gamma$ 's. Annihilations into two $\gamma$ 's will be separately calculated later on.

7 The mechanism has been also used in the model of Refs. [45, 51], explaining the monochromatic $\gamma$ at the Fermi LAT. But the light charged scalar faces a problem in explaining the neutrino mass.
} 


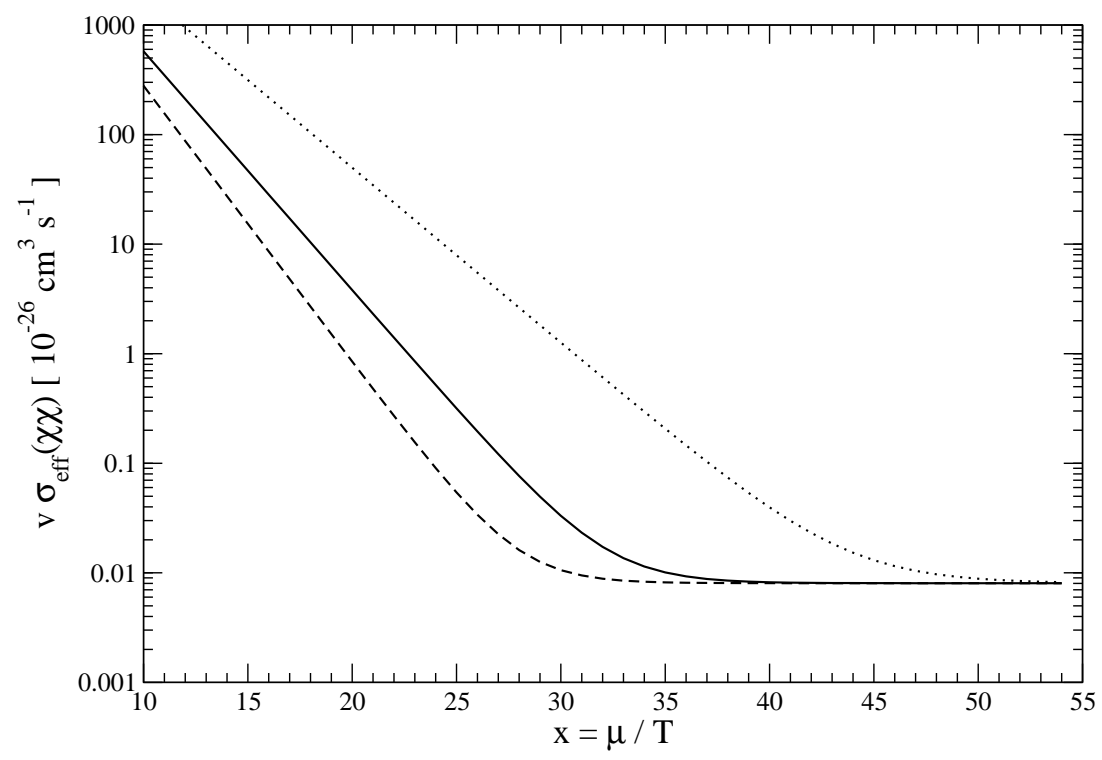

FIG. 5: The effective annihilation cross section [ the expression in [ ] of Eq. (35)] as a function of $x=\mu / T$ for $m_{\eta_{R}^{0}}=148$ (dotted), 153 (solid), and 156(dashed) GeV with $m_{\chi}$ fixed at $135 \mathrm{GeV}$.

[61]. In Fig. 5 we show the effective annihilation cross section [ the expression in [ ] of Eq. (35) ] as a function of $x=\mu / T$ for $m_{\eta_{R}^{0}}=148$ (dotted), 153 (solid), and 156 (dashed) $\mathrm{GeV}$, where we have fixed the parameters as

$$
\begin{aligned}
\lambda_{3} & =-1.26, \lambda_{4}=-0.0205, \gamma_{3}=11.3 \\
m_{\eta^{ \pm}} & =m_{\eta_{I}^{0}}=m_{\eta_{R}^{0}}+4 \mathrm{GeV}, m_{\chi}=135 \mathrm{GeV}, m_{h}=125 \mathrm{GeV} .
\end{aligned}
$$

As we see from Fig. 5 the effective cross section around the decoupling temperature $x \sim 20$ has a correct size and decreases drastically at low temperature. The effective cross section is normalized to $10^{-26} \mathrm{~cm}^{3} \mathrm{~s}^{-1}$, because it is the size to obtain the observed relic density of DM. The scalar couplings $\lambda_{2}, \gamma_{1}$ and $\gamma_{4}$ do not enter into the cross sections [ Eq. (32)], and $\gamma_{5}, \gamma_{6}, \gamma_{7}$ and $\kappa$ are irrelevant because $\phi$ is much heavier than $\eta$ and $\chi$.

In Fig. [6] we show the area in the $\gamma_{2}-\gamma_{3}$ plane in which the total relic density $\Omega_{T} h^{2}=$ $0.1157 \pm 0.0046(2 \sigma)$ with $m_{\chi}=135 \mathrm{GeV}$ can be obtained for $m_{\eta_{R}^{0}}=148$ (red), 152 (green), 153 (blue), 153.3 (cyan), 153.5 (purple), 154 (yellow) and 156 (black) GeV. The righthanded neutrino masses, $M_{k}$, are all $1 \mathrm{TeV}$, and the Yukawa couplings are chosen to yield $\sum_{i k}\left|Y_{i k}^{\nu}\right|^{2}=\left(10^{-4}\right)^{2}$. The vertical (black dashed) lines are the upper bounds of $\gamma_{2}$ set by the XENON 100 (right) and 1T (left) experiments [Eq. (30) ]. From Fig. 6] we can also see that there exists a parameter space with $m_{\chi}=135 \mathrm{GeV}, m_{\eta_{R}^{0}}>153 \mathrm{GeV}$, a large $\gamma_{3} / 4 \pi$ (between 0.65 and 1.0) and $\gamma_{2}$ satisfying the XENON constraint [Eq. (30) ]. A large $\gamma_{3}$ is needed to explain the $135 \mathrm{GeV} \gamma$-ray line in the Fermi spectrum, as we will see below. 


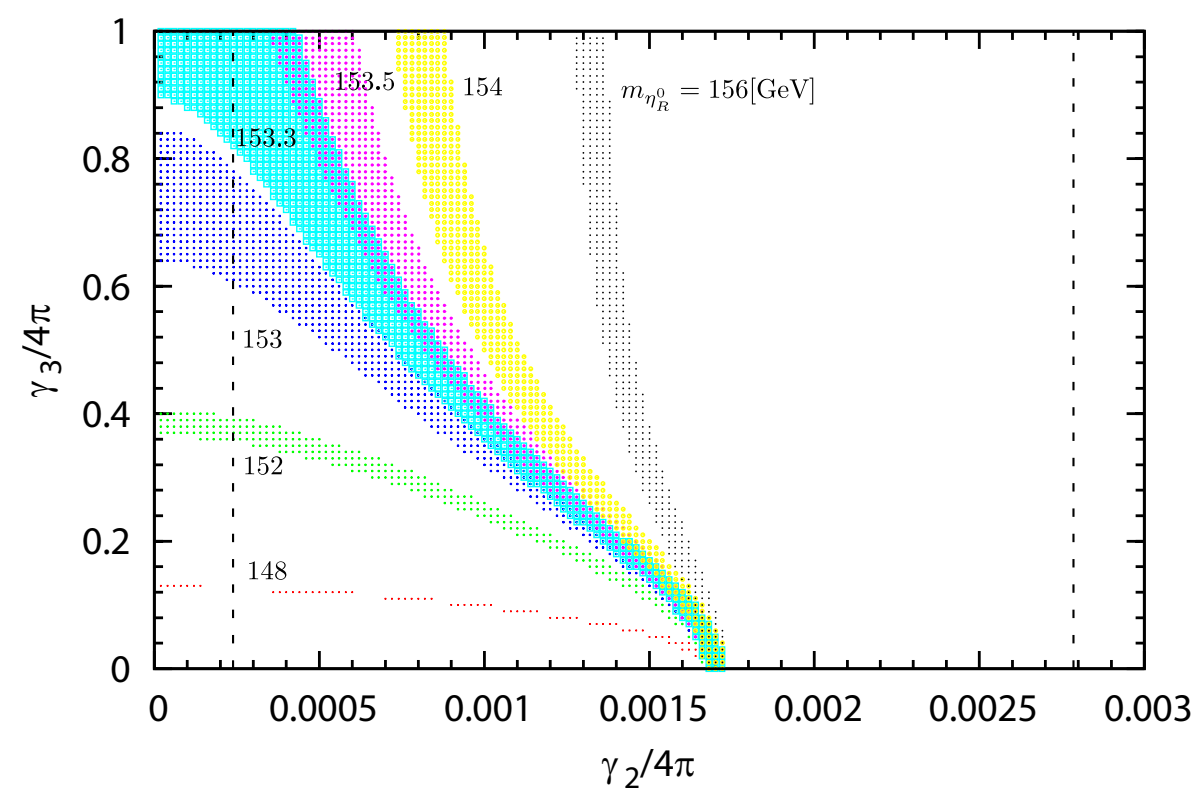

FIG. 6: The area with $\Omega_{T} h^{2}=0.1157 \pm 0.0046(2 \sigma)$ and $m_{\chi}=135 \mathrm{GeV}$ in the $\gamma_{2}-\gamma_{3}$ plane for $m_{\eta_{R}^{0}}=$ 148 (red), 152 (green), 153 (blue), 153.3 (cyan), 153.5 (purple), 154 (yellow) and 156 (black) GeV. The vertical (black dashed) lines are the upper bounds of $\gamma_{2}$ set by the XENON 100 (right) and 1T (left) experiments [Eq. (30)].

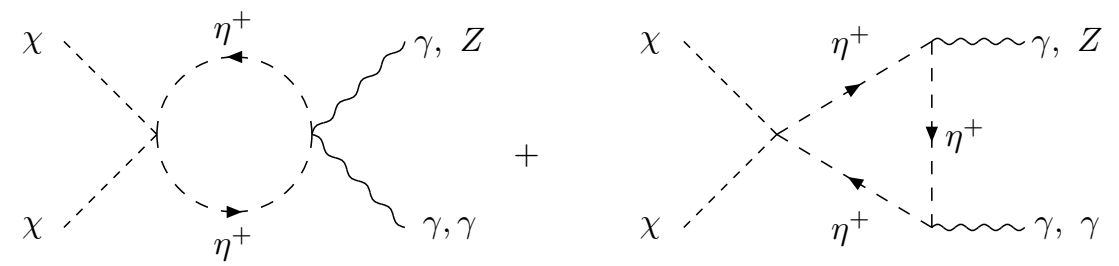

FIG. 7: One-loop diagrams for $\chi \chi \rightarrow \gamma \gamma$.

\section{Fermi LAT $135 \mathrm{GeV} \gamma$-ray line}

Our model contains the coupling between $\chi$ and $\eta^{+}\left(\mathcal{L}_{\eta^{\dagger} \eta \chi^{2}}=-\gamma_{3} \eta^{\dagger} \eta \chi^{2}\right)$. Because of this coupling there are diagrams in Fig. 17, which produce monochromatic $\gamma$ lines through the annihilation of $\chi$. We would like to use these diagrams to explain the monochromatic $\gamma$-ray line [36, 37, 40, 41] observed at the Fermi LAT [32 35]. There exist also s-channel diagrams with the SM Higgs propagator as shown in Fig. 8. The $h \chi \chi$ coupling is proportional to $\gamma_{2}$, while $h \eta_{R}^{0} \eta_{R}^{0}$ coupling is proportional to $\lambda_{3}$. The annihilation cross section $\sigma\left(\eta_{R}^{0} \eta_{R}^{0} \rightarrow \gamma \gamma\right)$ is 


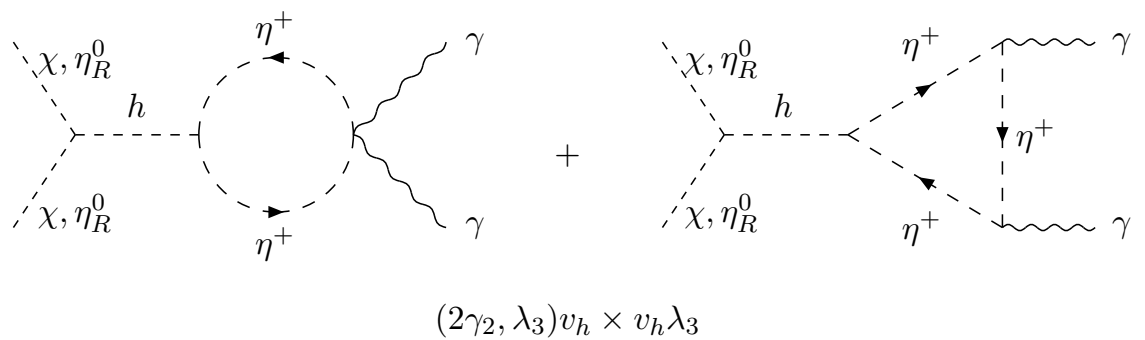

FIG. 8: S-channel diagrams for $\chi \chi, \eta_{R}^{0} \eta_{R}^{0} \rightarrow \gamma \gamma$.

large, because $\lambda_{3}$ is large [see Eqs. (26) and (27) $]^{8}$. Besides, due to the gauge interactions (see the right diagram of Fig. 4) the relic density of $\eta_{R}^{0}$ is very small, so the annihilation of the $\eta_{R}^{0}$ DM cannot contribute to the monochromatic $\gamma$-ray. Furthermore, for the same reason, the tree-level annihilations of $\eta_{R}^{0}$ into a pair of $W$ 's and $Z$ 's, which would contribute to the continuum $\gamma$-ray spectrum, are also suppressed. In contrast to this case, the pure gauge interaction (the right diagram of Fig. 4) is absent for the annihilation of $\chi$. The entire annihilations of $\chi$ into the SM particles are controlled by the single coupling $\gamma_{2}$, which has to satisfy the constraint of Eq. (30). Therefore, we may assume that the main contribution to $\sigma(\chi \chi \rightarrow \gamma \gamma)$ comes from the one-loop diagrams in Fig. 7 and find

$$
\begin{aligned}
\sigma(\chi \chi \rightarrow \gamma \gamma) v & =\frac{\gamma_{3}^{2} \alpha^{2} m_{\chi}^{2}}{32 \pi^{3} m_{\eta^{ \pm}}^{4}}\left|F_{0}\left(m_{\eta^{ \pm}}^{2} / m_{\chi}^{2}\right)\right|^{2} \\
& \simeq\left[\frac{\gamma_{3}}{4 \pi}\right]^{2}\left\{\begin{array}{l}
4.23 \\
2.66 \\
1.87 \\
1.39
\end{array} \times 10^{-27} \mathrm{~cm}^{3} \mathrm{~s}^{-1} \text { for } m_{\eta^{ \pm}}=\left\{\begin{array}{l}
140 \\
145 \\
150 \\
155
\end{array} \mathrm{GeV} .\right.\right.
\end{aligned}
$$

These values should be compared with $\left(1.27_{-0.43}^{+0.37}\right) \times 10^{-27} \mathrm{~cm}^{3} \mathrm{~s}^{-1}$ [37] for an Einasto DM galactic halo profile, which is the size that could explain the monochromatic $\gamma$ line observed at the Fermi LAT. So, if $\gamma_{3} / 4 \pi$ is large of $O(1)$, the desired value could be obtained. In Fig. 9 we show the area in the $m_{\eta^{ \pm}} \gamma_{3}$ plane in which $\sigma(\chi \chi \rightarrow \gamma \gamma) v=\left(1.27_{-0.43}^{+0.37}\right) \times 10^{-27} \mathrm{~cm}^{3} \mathrm{~s}^{-1}$ can be obtained. If $\eta^{ \pm}$is lighter than $\chi$, then $\chi$ can be annihilated into a pair of $\eta^{ \pm}$. For $m_{\chi}=135 \mathrm{GeV}$, the annihilation cross section becomes $\sigma\left(\chi \chi \rightarrow \eta^{+} \eta^{-}\right) v=\left(\gamma_{3}^{2} / 2 \pi m_{\chi}^{2}\right)(1-$ $\left.m_{\eta^{ \pm}}^{2} / m_{\chi}^{2}\right)^{1 / 2} \simeq \gamma_{3}^{2}\left(1-m_{\eta^{ \pm}}^{2} / m_{\chi}^{2}\right)^{1 / 2} \times 10^{-22} \mathrm{~cm}^{3} \mathrm{~s}^{-1}$, which is too large to obtain a sufficiently large relic density of $\chi$ for a large $\gamma_{3}$ unless $m_{\chi} \leq m_{\eta^{ \pm}}$. This is why we have to assume that $m_{\chi}<m_{\eta_{R}^{0}}, m_{\eta_{I}^{0}}, m_{\eta^{ \pm}}$. Comparing Fig. 9] with Fig. 6, we see that there is an overlapped area, that is, a parameter space in which $\sigma(\chi \chi \rightarrow \gamma \gamma) v \simeq 10 \times 10^{-27} \mathrm{~cm}^{3} \mathrm{~s}^{-1}$ and $\Omega_{T} h^{2} \simeq 0.12$ can

\footnotetext{
${ }^{8}$ In [42], $\sigma\left(\eta_{R}^{0} \eta_{R}^{0} \rightarrow \gamma \gamma\right)$ is used to explain the monochromatic $\gamma$-ray line, where a fine-tuned cancellation mechanism to suppress the total annihilation cross section of $\eta_{R}^{0}[24]$ is employed
} 


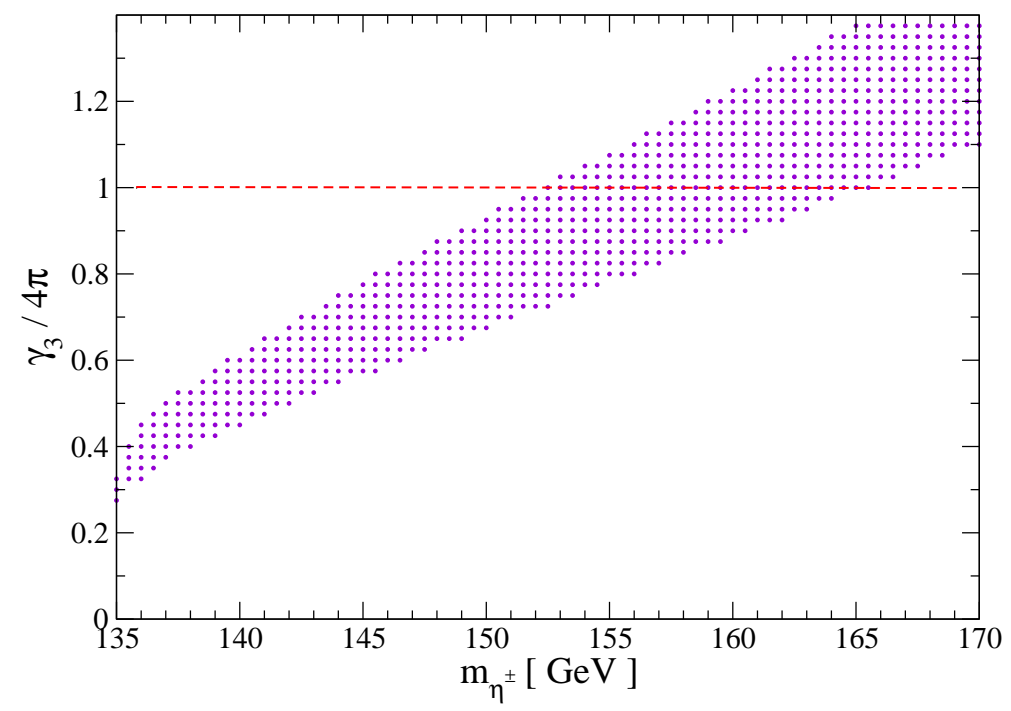

FIG. 9: The area for $\sigma(\chi \chi \rightarrow \gamma \gamma) v=\left(1.27_{-0.43}^{+0.37}\right) \times 10^{-27} \mathrm{~cm}^{3} \mathrm{~s}^{-1}$ in the $m_{\eta^{ \pm}}-\gamma_{3}$ plane.

be obtained. This is shown in Fig. 10 for $m_{\eta_{R}^{0}}=153.3 \mathrm{GeV}$ and $m_{\eta^{ \pm}}=m_{\eta_{R}^{0}}+4 \mathrm{GeV}=157.3$ $\mathrm{GeV}$.

The same diagrams as in Fig. 7 also produce $\gamma Z$. The annihilation cross section is given by

$$
\sigma(\chi \chi \rightarrow \gamma Z) v=\frac{\gamma_{3}^{2} \alpha^{2} m_{\chi}^{2} \cot ^{2}\left(2 \theta_{W}\right)}{32 \pi^{3} m_{\eta^{ \pm}}^{4}}\left|\hat{F}_{0}\left(m_{\eta^{ \pm}}^{2} / m_{\chi}^{2}\right)\right|^{2}\left(1-\frac{m_{Z}^{2}}{4 m_{\chi}^{2}}\right)
$$

where

$$
\hat{F}_{0}\left(\tau_{\eta}\right)=\tau_{\eta}\left[\frac{1}{2}-\int_{0}^{1} d x \int_{0}^{1-x} d y \frac{\tau_{\eta}-\tau_{Z}\left(x^{2}+y^{2}\right)+4 x y\left(1-\tau_{Z} / 2\right)}{\tau_{\eta}+\tau_{Z}\left(x^{2}+y^{2}\right)-4 x y\left(1-\tau_{Z} / 2\right)}\right]
$$

with $\tau_{\eta}=m_{\eta^{ \pm}}^{2} / m_{\chi}^{2}$ and $\tau_{Z}=m_{Z}^{2} / m_{\chi}^{2}$. For $m_{\eta^{ \pm}}=150 \mathrm{GeV}$ and $m_{\chi}=135 \mathrm{GeV}$, for instance, we obtain $\sigma(\chi \chi \rightarrow \gamma Z) v=3.5\left(\gamma_{3} / 4 \pi\right)^{2} \times 10^{-29} \mathrm{~cm}^{3} \mathrm{~s}^{-1}$, which is about $2 \%$ of $\sigma(\chi \chi \rightarrow \gamma \gamma) v$. According to Ref. [64], this is welcome to explain the Fermi LAT monochromatic $\gamma$ line.

We also have to satisfy the constraints on the continuum $\gamma$ and follow the analyses of Ref. [64] (see also Refs. [65, 66], which give similar constraints). They consider two different constraints, supersaturation constraint and shape constraint, which can be transferred to the upper bound on the ratio of the total annihilation cross section to $\sigma(\chi \chi \rightarrow \gamma \gamma)$. Specifically, they consider the constraint on the theoretical ratio

$$
R_{T}^{\mathrm{th}}=\frac{\sigma_{T}}{2 \sigma_{\gamma \gamma}+\sigma_{\gamma Z}}
$$

where $\sigma_{T}$ is the total annihilation cross section. Since the dominant origins for the continuum $\gamma$ are $\sigma\left(\chi \chi \rightarrow W^{+} W^{-}, Z Z, b \bar{b}, \tau^{+} \tau^{-}, \mu^{+} \mu^{-}\right)$, we also consider the individual cross sections and calculate

$$
R_{\alpha}^{\mathrm{th}}=\frac{\sigma(\chi \chi \rightarrow \alpha)}{2 \sigma(\chi \chi \rightarrow \gamma \gamma)+\sigma(\chi \chi \rightarrow \gamma Z)} \simeq \frac{\sigma(\chi \chi \rightarrow \alpha)}{2 \sigma(\chi \chi \rightarrow \gamma \gamma)}
$$




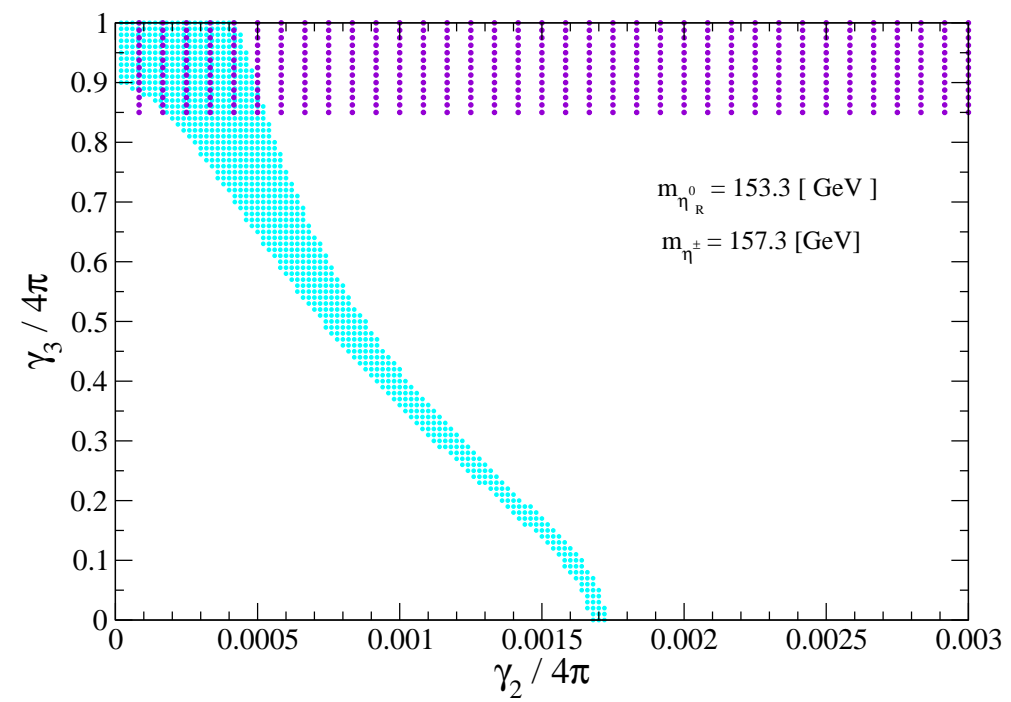

FIG. 10: The parameter space (overlapped area) in which $\sigma(\chi \chi \rightarrow \gamma \gamma) v=\left(1.27_{-0.43}^{+0.37}\right) \times 10^{-27} \mathrm{~cm}^{3} \mathrm{~s}^{-1}$ and $\Omega_{T} h^{2}=0.1157 \pm 0.0046(2 \sigma)$ can be obtained for $m_{\eta_{R}^{0}}=153.3 \mathrm{GeV}$ and $m_{\eta^{ \pm}}=m_{\eta_{R}^{0}}+4 \mathrm{GeV}=157.3 \mathrm{GeV}$.

in addition to $R_{\mathrm{SM}}^{\mathrm{th}} \simeq \sigma(\chi \chi \rightarrow \mathrm{SM}) / 2 \sigma(\chi \chi \rightarrow \gamma \gamma)$, where $\alpha=W^{+} W^{-}, Z Z, f \bar{f}$. We use the same parameter values given in Eq. (37) with $\gamma_{2} / 4 \pi=1.0 \times 10^{-4}$ and $m_{\eta_{R}^{0}}=153.3 \mathrm{GeV}$, and we obtain

$$
\Omega_{\eta} h^{2}=0.981 \times 10^{-5}, \Omega_{\chi} h^{2}=0.1197, \Omega_{T} h^{2}=0.1197
$$

and

$$
\begin{aligned}
\sigma(\chi \chi \rightarrow \gamma \gamma) v & \simeq 1.2 \times 10^{-27} \mathrm{~cm}^{3} \mathrm{~s}^{-1}, \\
\sigma(\chi \chi \rightarrow \mathrm{SM}) v & \simeq 8.0 \times 10^{-29} \mathrm{~cm}^{3} \mathrm{~s}^{-1}, R_{\mathrm{SM}}^{\mathrm{th}} \simeq 0.06 \\
\sigma\left(\chi \chi \rightarrow W^{+} W^{-}\right) v & \simeq 3.9 \times 10^{-29} \mathrm{~cm}^{3} \mathrm{~s}^{-1}, R_{W}^{\mathrm{th}} \simeq 0.03 \\
\sigma(\chi \chi \rightarrow Z Z) v & \simeq 1.7 \times 10^{-29} \mathrm{~cm}^{3} \mathrm{~s}^{-1}, R_{Z}^{\mathrm{th}} \simeq 0.01 \\
\sigma(\chi \chi \rightarrow h h) v & \simeq 2.5 \times 10^{-29} \mathrm{~cm}^{3} \mathrm{~s}^{-1}, R_{h}^{\mathrm{th}} \simeq 0.02 \\
\sigma(\chi \chi \rightarrow f \bar{f}) v & \simeq 1.1 \times 10^{-31} \mathrm{~cm}^{3} \mathrm{~s}^{-1}, R_{f}^{\mathrm{th}} \simeq 10^{-4}
\end{aligned}
$$

These values of $R^{\text {th }}$ should satisfy the supersaturation constraint as well as the shape constraint of Ref. [64] (see also Ref. [65]). There are also constraints coming from the antiprotonto-proton flux observed by the PAMELA [67]. Antiprotons can be produced by the DM annihilations into the gauge bosons, Higgs bosons and quarks. To explain the PAMELA data, these productions have to be suppressed. The annihilation cross sections $\times v$ given in Eqs. (45)-(49) satisfy all the constraints, including the most stringent one, $\lesssim 10^{-26} \mathrm{~cm}^{3} \mathrm{~s}^{-1}$ [64 66, 68 71]. So, the model could explain the monochromatic $\gamma$ line observed at the Fermi LAT if $m_{\eta^{ \pm}} \simeq 153 \mathrm{GeV}$ and $\gamma_{3} / 4 \pi \sim O(1)$, which is at the border of perturbation theory.

Therefore, in this parameter space the model has a meaning only up to a scale slightly above the electroweak scale, and the scalar potential is stable only below that energy. In 
this sense, the SM is more natural in the parameter space in which the model has a potential to explain the $\gamma$ excess both in the Higgs decay and at the Fermi LAT. In other parameter space, where the scalar couplings are small but the $\gamma$ excesses cannot be (or do not have to be) simultaneously explained, the model can remain well defined to a very high energy close to the Planck scale. This situation would occur if the monochromatic $\gamma$ ray in the Fermi data turn out to be an instrumental effect (see for instance Refs. [72, 73]). Then $\gamma_{3}$ does not have to be large, and the $\chi$ DM does not have to be lighter than the $\eta \mathrm{DM}$, so that, to explain the diphoton mode in the Higgs decay, the scalar coupling $\lambda_{3}$ can become much smaller (because the decay mode can be enhanced by a smaller mass of the charged

$\left.\eta^{ \pm}[30,31]\right)$. In this parameter space, the model can remain perturbative for a wide range of energy scales.

\section{CONCLUSION AND DISCUSSION}

In this paper we have proposed a non-supersymmetric model of a two-loop radiative seesaw, in which the lepton number is softly broken by a dimension-two operator, and the tree-level Dirac mass is forbidden by $Z_{2} \times Z_{2}^{\prime}$. This discrete symmetry can be used to stabilize two or three dark matter particles. The model contains, in addition to the SM Higgs field, an inert $S U(2)_{L}$ doublet scalar $\eta$ and two inert singlet scalars $\phi$ and $\chi$, and this is a minimal set. We have considered the SM Higgs boson decay into two $\gamma^{\prime}$ s and found that it is enhanced by $\eta^{+}$circulating in one-loop diagrams for $h \rightarrow \gamma \gamma . \eta^{+}$is also circulating in similar one-loop diagrams contributing to $\chi \chi \rightarrow \gamma \gamma$, and we have found that the model has a potential to explain the Fermi LAT $135 \mathrm{GeV} \gamma$-ray line.

The mechanism to explain the Fermi LAT $135 \mathrm{GeV} \gamma$-ray line in the present model is strongly based on the fact that there exist some particles of similar masses where at least one of them is DM. Let us briefly outline the mechanism [50, 51]. Annihilation (or decay) of DM into $\gamma$ 's happens always at the loop level. Those into the SM particles, i.e. $W, Z$, Higgs boson pairs, etc., are usually possible at the tree level, and they produce continuum $\gamma$ rays as well as antiprotons. To explain the $135 \mathrm{GeV} \gamma$-ray line, we have to suppress these tree-level processes somehow, or enhance the loop process, while keeping the relic abundance of DM at the observed value. In the present model this is realized in the following way: There are two kinds of the tree-level DM annihilations; one into the SM particles and the other into a pair of other DM particles (DM conversion). The slightly heavier DM ( $\eta_{R}^{0}$ in our model) has large annihilation cross sections both into the SM particles and other DM particles, so its relic density is very small. The $135 \mathrm{GeV} \gamma$-ray line comes mainly from the annihilation of the slightly lighter DM ( $\chi$ in our model). Its annihilation cross section into the SM particles has to be sufficiently small to suppress the continuum $\gamma$ rays and the production of antiprotons. Although the annihilation of the lighter DM into heavier DM is kinematically forbidden at zero temperature, this process becomes operative at high temperature: The smaller the 
mass difference of two DM particles is, the more effective is the DM conversion. So, at high temperature the annihilation of the lighter DM is controlled by the mass deference and can be large, but at low temperature, this conversion process practically disappears (see Fig. 51). (For this mechanism to work, the slightly heavier particle does not have to be stable. ) It is, however, important that the lighter one be SM gauge singlet to avoid the fact that the tree-level annihilations are entirely controlled by the SM gauge interactions. (In the present model, the $\chi \mathrm{DM}$ is not ad hoc introduced.) This is the reason why we can obtain the observed value of the relic density for the lighter DM, although the annihilation cross sections of the lighter DM into the SM particles are very small $\lesssim O\left(10^{-27}\right) \mathrm{cm}^{3} \mathrm{~s}^{-1}$ in the galaxy.

The annihilation cross sections into the SM particles given in Eqs. (43), (45)-(49) are obtained without one-loop corrections. Strictly speaking, we should include the one-loop corrections, because the tree-level contributions are so small that the one-loop corrections may be larger than the tree-level corrections. Similarly, the relic densities for Fig. 6 and also Eq. (43) have been computed by neglecting the coannihilation of the DM particles with the charged and CP-odd components of $\eta$, although we have assumed that their mass differences are not large [i.e. $m_{\eta^{ \pm}}-m_{\chi}=(17-25) \mathrm{GeV}, m_{\eta^{ \pm}}-m_{\eta_{R}^{0}}=4 \mathrm{GeV}$ and $m_{\eta^{ \pm}}=m_{\eta_{I}^{0}}$. The one-loop corrections would change $\gamma_{2}$ effectively. That is, one-loop corrections can be partially absorbed into $\gamma_{2}$, so that the annihilation cross sections [ Eq. (45)-(49)] would change only slightly; to transgress the supersaturation constraint and shape constraint, a change of 2 orders of magnitude is needed. The coannihilations also would effectively increase $\gamma_{3}$. To obtain a realistic relic abundance for the $\chi \mathrm{DM}$ in this situation, the mass of the $\eta$ DM mass should be slightly increased, as one can see from Fig. 6. The one-loop analysis including the coannihilations is beyond the scope of the present paper, and we will leave it for our future project.

We have assumed throughout that the $\phi$ is so heavy that it decays into a $\eta_{R}^{0}$ and a $\chi$. In the case that $m_{\phi_{R}}<m_{\chi}+m_{\eta_{R}^{0}}$, it becomes the third DM, whose annihilation may be responsible for the second monochromatic $\gamma$-ray line in the Fermi data [39]. We leave this question to the future program.

We thank Michael Duerr, Maria Krawczyk and Abdesslam Arhrib for useful discussions. M. A. thanks the Max-Planck-Institut für Kernphysik, Heidelberg for kind hospitality. The work of M. A. is supported in part by the Grant-in-Aid for Scientific Research for Young Scientists (B) (Grant No. 22740137), and J. K. is partially supported by the Grant-in-Aid for Scientific Research (C) from the Japan Society for Promotion of Science (Grant No. 22540271).

[1] P. Minkowski, Phys. Lett. B 67 (1977) 421. 
[2] M. Gell-Mann, P. Ramond, and R. Slansky, in Supergravity, eds. P. van Nieuwenhuizen and D. Z. Freedman (North-Holland, 1979), p. 315; T. Yanagida, in Proc. of the Workshop on the Unified Theory and the Baryon Number in the Universe, eds. O. Sawada and A. Sugamoto, KEK Report No. 79-18 (Tsukuba, Japan, 1979), p. 95.

[3] R. N. Mohapatra and G. Senjanovic, Phys. Rev. Lett. 44 (1980) 912.

[4] A. Zee, Phys. Lett. B 93 (1980) 389 [Erratum-ibid. B 95 (1980) 461].

[5] A. Zee, Nucl. Phys. B 264 (1986) 99.

[6] K. S. Babu, Phys. Lett. B 203 (1988) 132.

[7] L. M. Krauss, S. Nasri and M. Trodden, Phys. Rev. D 67 (2003) 085002 hep-ph/0210389.

[8] E. Ma, Phys. Rev. D 73 (2006) 077301 hep-ph/0601225.

[9] K. Cheung and O. Seto, Phys. Rev. D 69 (2004) 113009 hep-ph/0403003.

[10] J. Kubo, E. Ma and D. Suematsu, Phys. Lett. B 642 (2006) 18 hep-ph/0604114.

[11] M. Aoki, S. Kanemura and O. Seto, Phys. Rev. Lett. 102 (2009) 051805 arXiv:0807.0361 [hep-ph]]; Phys. Rev. D 80 (2009) 033007 arXiv:0904.3829 [hep-ph]].

[12] F. Bonnet, M. Hirsch, T. Ota and W. Winter, JHEP 1207 (2012) 153 arXiv:1204.5862 [hep$\mathrm{ph}]$.

[13] Y. Farzan, S. Pascoli and M. A. Schmidt, JHEP 1303 (2013) 107 [arXiv:1208.2732 [hep-ph]].

[14] S. Kanemura and T. Ota, Phys. Lett. B 694 (2010) 233 [arXiv:1009.3845 [hep-ph]].

[15] S. Kanemura, T. Nabeshima and H. Sugiyama, Phys. Lett. B 703 (2011) 66 [arXiv:1106.2480 [hep-ph]].

[16] S. S. C. Law and K. L. McDonald, Phys. Lett. B 713 (2012) 490 arXiv:1204.2529 [hep-ph]].

[17] G. Guo, X. -G. He and G. -N. Li, JHEP 1210 (2012) 044 [arXiv:1207.6308 [hep-ph]].

[18] F. D'Eramo and J. Thaler, JHEP 1006 (2010) 109 [arXiv:1003.5912 [hep-ph]].

[19] G. Belanger and J.-C. Park, JCAP 1203 (2012) 038 arXiv:1112.4491 [hep-ph]].

[20] G. Belanger, K. Kannike, A. Pukhov and M. Raidal, JCAP 1204 (2012) 010 arXiv:1202.2962 [hep-ph]].

[21] M. Aoki, M. Duerr, J. Kubo and H. Takano, Phys. Rev. D 86 (2012) 076015 arXiv:1207.3318 [hep-ph]].

[22] L. Lopez Honorez, E. Nezri, J. F. Oliver and M. H. G. Tytgat, JCAP 0702 (2007) 028 arXiv:hep-ph/0612275.

[23] E. M. Dolle and S. Su, Phys. Rev. D 80 (2009) 055012 arXiv:0906.1609 [hep-ph]].

[24] L. Lopez Honorez and C. E. Yaguna, JCAP 1101 (2011) 002 [arXiv:1011.1411 [hep-ph]].

[25] G. Aad et al. [ATLAS Collaboration], Phys. Lett. B 716 (2012) 1 [arXiv:1207.7214 [hep-ex]].

[26] S. Chatrchyan et al. [CMS Collaboration], Phys. Lett. B 716 (2012) 30 arXiv:1207.7235 [hep-ex]].

[27] ATLAS Collaboration, ATLAS-CONF-2012-091.

[28] J. F. Gunion, H. E. Haber, G. L. Kane and S. Dawson, Front. Phys. 80 (2000) 1.

[29] A. G. Akeroyd, M. A. Diaz and M. A. Rivera, Phys. Rev. D 76 (2007) 115012 arXiv:0708.1939 
[hep-ph]].

[30] A. Arhrib, R. Benbrik and N. Gaur, Phys. Rev. D 85 (2012) 095021 [arXiv:1201.2644 [hep-ph]].

[31] B. Swiezewska and M. Krawczyk, arXiv:1212.4100 [hep-ph].

[32] W. B. Atwood et al. [LAT Collaboration], Astrophys. J. 697 (2009) 1071 arXiv:0902.1089 [astro-ph.IM]].

[33] A. A. Abdo, M. Ackermann, M. Ajello, W. B. Atwood, L. Baldini, J. Ballet, G. Barbiellini and D. Bastieri et al., Phys. Rev. Lett. 104 (2010) 091302 arXiv:1001.4836 [astro-ph.HE]].

[34] M. Ackermann et al. [LAT Collaboration], Phys. Rev. D 86 (2012) 022002 [arXiv:1205.2739 [astro-ph.HE]].

[35] Fermi-LAT Collaboration, http://fermi.gsfc.nasa.gov/science/mtgs/symposia/2012/program/fri/AAlbert

[36] T. Bringmann, X. Huang, A. Ibarra, S. Vogl and C. Weniger, JCAP 1207 (2012) 054 arXiv:1203.1312 [hep-ph]].

[37] C. Weniger, JCAP 1208 (2012) 007 [arXiv:1204.2797 [hep-ph]].

[38] E. Tempel, A. Hektor and M. Raidal, JCAP 1209, 032 (2012) [Addendum-ibid. 1211, A01 (2012)] arXiv:1205.1045 [hep-ph]].

[39] A. Rajaraman, T. M. P. Tait and D. Whiteson, JCAP 1209 (2012) 003 arXiv:1205.4723 [hep-ph]].

[40] M. Su and D. P. Finkbeiner, arXiv:1206.1616 [astro-ph.HE].

[41] M. Su and D. P. Finkbeiner, arXiv:1207.7060 [astro-ph.HE].

[42] A. Biswas, D. Majumdar, A. Sil and P. Bhattacharjee, arXiv:1301.3668 [hep-ph].

[43] F. D'Eramo, M. McCullough and J. Thaler, JCAP 1304 (2013) 030 arXiv:1210.7817 [hep$\mathrm{ph}]]$.

[44] P. -H. Gu, arXiv:1301.4368 [hep-ph].

[45] S. Baek, P. Ko and E. Senaha, arXiv:1209.1685 [hep-ph].

[46] Y. Bai, V. Barger, L. L. Everett and G. Shaughnessy, arXiv:1212.5604 [hep-ph].

[47] J. M. Cline, Phys. Rev. D 86 (2012) 015016 [arXiv:1205.2688 [hep-ph]].

[48] L. Wang and X. -F. Han, Phys. Rev. D 87 (2013) 015015 [arXiv:1209.0376 [hep-ph]].

[49] J. Fan and M. Reece, arXiv:1301.2597 [hep-ph].

[50] K. Griest, D. Seckel, Phys. Rev. D43 (1991) 3191-3203.

[51] S. Tulin, H. -B. Yu, K. M. Zurek and , Phys. Rev. D 87 (2013) 036011 arXiv:1208.0009 [hep-ph]].

[52] E. Ma, Phys. Lett. B 659 (2008) 885 [arXiv:0710.2325 [hep-ph]].

[53] W. A. Bardeen, FERMILAB-CONF-95-391-T.

[54] H. Aoki, S. Iso and, Phys. Rev. D 86 (2012) 013001 [arXiv:1201.0857 [hep-ph]].

[55] G. Passarino and M. J. G. Veltman, Nucl. Phys. B 160, 151 (1979).

[56] E. Ma and M. Raidal, Phys. Rev. Lett. 87 (2001) 011802 [Erratum-ibid. 87 (2001) 159901] arXiv:hep-ph/0102255.

[57] K. Hayasaka, arXiv:1010.3746 [hep-ex]. 
[58] J. Beringer et al. [Particle Data Group Collaboration], Phys. Rev. D 86 (2012) 010001.

[59] R. Barbieri, L. J. Hall and V. S. Rychkov, Phys. Rev. D 74 (2006) 015007 arXiv:hep-ph/0603188.

[60] J. R. Ellis, A. Ferstl and K. A. Olive, Phys. Lett. B 481 (2000) 304 arXiv:hep-ph/0001005].

[61] G. Hinshaw, D. Larson, E. Komatsu, D. N. Spergel, C. L. Bennett, J. Dunkley, M. R. Nolta and M. Halpern et al., arXiv:1212.5226 [astro-ph.CO].

[62] E. Aprile et al. [XENON100 Collaboration], Phys. Rev. Lett. 109 (2012) 181301 arXiv:1207.5988 [astro-ph.CO]].

[63] E. Aprile [XENON1T Collaboration], arXiv:1206.6288 [astro-ph.IM].

[64] T. Cohen, M. Lisanti, T. R. Slatyer and J. G. Wacker, JHEP 1210 (2012) 134 arXiv:1207.0800 [hep-ph]].

[65] W. Buchmuller and M. Garny, JCAP 1208 (2012) 035 arXiv:1206.7056 [hep-ph]].

[66] G. Belanger, C. Boehm, M. Cirelli, J. Da Silva and A. Pukhov, JCAP 1211, 028 (2012) arXiv:1208.5009 [hep-ph]].

[67] O. Adriani et al. [PAMELA Collaboration], Phys. Rev. Lett. 105 (2010) 121101 arXiv:1007.0821 [astro-ph.HE]].

[68] A. Ibarra and D. Tran, JCAP 0807 (2008) 002 arXiv:0804.4596 [astro-ph]].

[69] F. Donato, D. Maurin, P. Brun, T. Delahaye and P. Salati, Phys. Rev. Lett. 102 (2009) 071301 arXiv:0810.5292 [astro-ph]].

[70] W. Buchmuller, A. Ibarra, T. Shindou, F. Takayama and D. Tran, JCAP 0909 (2009) 021 arXiv:0906.1187 [hep-ph]].

[71] M. Asano, T. Bringmann, G. Sigl and M. Vollmann, Phys. Rev. D 87 (2013) 103509 arXiv:1211.6739 [hep-ph]].

[72] D. Whiteson, arXiv:1302.0427 [astro-ph.HE].

[73] C. Weniger, arXiv:1303.1798 [astro-ph.HE]. 